\title{
PPM1A as a key target of the application of Jiawei-Maxing-Shigan decoction for the attenuation of radiation-induced epithelial-mesenchymal transition in type II alveolar epithelial cells
}

\author{
JINHUA LU ${ }^{1 *}$, SHENGYOU LIN ${ }^{2 *}$, ZECHEN LIN $^{1}$, XIANLEI LIN ${ }^{1}$, \\ YUEZHONG SHEN ${ }^{3}$ and JINGYANG SU ${ }^{3}$

\begin{abstract}
${ }^{1}$ Oncology Department, Dingqiao Branch of GuangXing Hospital Affiliated to Zhejiang Chinese Medical University;
${ }^{2}$ Oncology Department, GuangXing Hospital Affiliated to Zhejiang Chinese Medical University;

${ }^{3}$ Graduate School, Zhejiang Chinese Medical University, Hangzhou, Zhejiang 310007, P.R. China
\end{abstract}

Received March 18, 2021; Accepted July 26, 2021

DOI: $10.3892 / \mathrm{mmr} .2021 .12465$

\begin{abstract}
Radiation-induced lung tissue injury is an important reason for the limited application of radiotherapy on thoracic malignancies. Previously, we reported that administration of Jiawei-Maxing-Shigan decoction (JMSD) attenuated the radiation-induced epithelial-mesenchymal transition (EMT) in alveolar epithelial cells (AECs) via TGF- $\beta /$ Smad signaling. The present study aimed to examine the role of protein phosphatase $\mathrm{Mg}^{2+} / \mathrm{Mn}^{2+}$-dependent 1A (PPM1A) in the anti-EMT activity of JMSD on AECs. The components in the aqueous extract of JMSD were identified by high-performance liquid chromatography coupled with electrospray mass spectrometry. Primary rat type II AECs were treated with radiation (60Co $\gamma$-ray at $8 \mathrm{~Gy}$ ) and JMSD-medicated serum. PPM1A was overexpressed and knocked down in the AECs via lentivirus transduction and the effects of JMSD administration on the key proteins related to TGF- $\beta 1 / \mathrm{Smad}$ signaling were measured by western blotting. It was found that radiation decreased the PPM1A expression in the AECs and JMSD-medicated serum upregulated the PPM1A expressions in the radiation-induced AECs. PPM1A overexpression increased the E-cadherin level but decreased the phosphorylated (p-)Smad2/3, vimentin and $\alpha$-smooth muscle actin ( $\alpha$-SMA) levels in the AECs. By contrast, the PPM1A knockdown decreased the E-cadherin level and increased the $\mathrm{p}$-Smad2/3, vimentin and $\alpha$-SMA levels
\end{abstract}

Correspondence to: Dr Shengyou Lin, Oncology Department, GuangXing Hospital Affiliated to Zhejiang Chinese Medical University, 453 Tiyuchang Road, Xihu, Hangzhou, Zhejiang 310007, P.R. China

E-mail: linsy0628@126.com

*Contributed equally

Key words: protein phosphatase $\mathrm{Mg}^{2+} / \mathrm{Mn}^{2+}$-dependent 1A, Jiawei-Maxing-Shigan decoction, epithelial-mesenchymal transition, Radiation-induced injury, TGF- $\beta 1 /$ Smad signaling in the AECs and these effects could be blocked by SB431542 (TGF- $11 /$ Smad signaling inhibitor). JMSD administration increased the E-cadherin level and decreased the p-Smad2/3, vimentin and $\alpha$-SMA levels in the AECs; however, these effects could be blocked by siPPM1A-2. In conclusion, PPM1A is a key target of JMSD administration for the attenuation of the radiation-induced EMT in primary type II AECs via the TGF- $\beta 1 /$ Smad pathway.

\section{Introduction}

Currently, besides surgery and chemotherapy, radiotherapy remains one of the most commonly used treatment methods for thoracic malignant tumors such as lung cancer, breast cancer, neck cancer and lymphoma $(1,2)$. Radiation-induced normal lung tissue injury is a common complication of chest radiotherapy and is an important reason for the limited application of radiotherapy in patients with thoracic malignancies $(3,4)$. Main radiation-induced injury is reported to result in acute pneumonitis and, later, fibrosis in lung tissue (4). In addition, studies have indicated that radiation could result in injury of alveolar epithelial cells (AECs) and subsequently induce epithelial-mesenchymal transition (EMT) of AECs. This is one of the possible pathological mechanisms of pulmonary fibrosis after radiotherapy $(5,6)$. Therefore, suppression of the EMT process in AECs would be beneficial for controlling or preventing the development of pulmonary fibrosis under radiotherapy.

Jiawei-Maxing-Shigan decoction (JMSD), is composed of Roasted ephedra (Herba Ephedrae), Gypsum (Gypsum Fibrosum), Paeoniae radix (Radix Paeoniae Rubra), Apricot seed (Semen Armeniacae Amarum), Mulberry (Cortex mori Radicis), Honeysuckle (Flos Lonicerae Japonicae) and Licorice (Radix Et Rhizoma Glycyrrhizae) (7) and is a clinically proven recipe for treating pediatric asthma (8), bronchitis (9), infantile mycoplasma pneumonia (10) and radioactive lung injury $(7,11)$. Our previous study found that the main constituents of JMSD are farnesene, dihydrotanshinone I, paeonol, emodin, schisanhenol, tanshinone IIA, cryptotanshinone, 
columbianadin, uridine and liquiritigenin (7). Furthermore, JMSD administration can attenuate radiation-induced EMT in AECs by regulating the expression of TGF- $\beta / \mathrm{Smad}$ signaling (7). Protein phosphatase $\mathrm{Mg}^{2+} / \mathrm{Mn}^{2+}$-dependent $1 \mathrm{~A}$ (PPM1A) serves an important role in the signal transduction of TGF- $\beta /$ Smad signaling and can inactivate TGF- $\beta / \mathrm{Smad}$ signaling by dephosphorylating Smad2/3. PPM1A functions as a tumor suppressor in bladder and breast cancer via regulating cell invasion, EMT and cell cycle progression (12-14). As part of a continuing study on the molecular mechanisms of JMSD, the present study further examined the role of PPM1A in the anti-EMT activity of JMSD on AECs.

\section{Materials and methods}

Herbal medicines. The seven herbal medicines were supplied and the JMSD water extract was prepared by the School of Pharmacy, Zhejiang Chinese Medicine University. The composition of JMSD is presented in Table I. The seven herbs were decocted by boiling in distilled water for $1 \mathrm{~h}$ twice as described in our previous paper (7). Then, the water extract was filtered and concentrated to $32 \mathrm{ml}$. As JMSD was a mixture, its purity could not be determined.

Liquid chromatography coupled with electrospray mass spectrometry (HPLC/ESI-MS) analysis of JMSD. The aqueous extract of JMSD was analyzed with an Agilent 1100 HPLC system (Agilent Technologies, Inc.) coupled with electrospray mass spectrometry as previously described (7). The separation was performed on a GS-120-5-C18-BIO chromatographic column $(5 \mu \mathrm{m} ; 250 \times 4.6 \mathrm{~mm}$ i.d.; Global Chromatography Co., Ltd.) with the column temperature set at $35^{\circ} \mathrm{C}$. A linear gradient elution of A ( $0.1 \%$ formic acid water) and B (acetonitrile) was used with the gradient procedure as follows: B 5\% at $0 \mathrm{~min}, \mathrm{~B} 40 \%$ at $60 \mathrm{~min}(\mathrm{v} / \mathrm{v})$. The flow rate was $1.0 \mathrm{ml} / \mathrm{min}$ and the injection volume was $10 \mu \mathrm{l}$. DAD was on and the target wavelength was simultaneously set at $210 \mathrm{~nm}$. The split ratio to the mass spectrometer was 1:3. The acquisition parameters for negative ion mode were: collision gas, ultra high-purity helium (He), nebulizer gas (N2), 35 psi, drying gas (N2), $10 \mathrm{l} / \mathrm{min}$, drying temperature, $350^{\circ} \mathrm{C}, \mathrm{HV}, 3500 \mathrm{~V}$, mass scan range, $\mathrm{m} / \mathrm{z} 100-2200$, target mass, $500 \mathrm{~m} / \mathrm{z}$, compound stability, $100 \%$, trap drive level, $100 \%$. All the data were analyzed using the Chemstation software (version B.04.03; Agilent Technologies, Inc.).

Animals. Male Sprague-Dawley (SD) rats (6-8 weeks; $200 \pm 20 \mathrm{~g} ; \mathrm{n}=3$ ) and Wistar rats (6-8 weeks; $200 \pm 20 \mathrm{~g} ; \mathrm{n}=6$ ) were acquired from the Shanghai Experimental Animal Center (Shanghai, China). Rats were maintained at $25^{\circ} \mathrm{C}$ with $60 \%$ humidity and a 12-h light/dark cycle, and free access to food and water. The experimental protocols were approved by the animal ethics committee of Zhejiang Chinese Medicine University (Hangzhou, China; approval no. 2019485).

Chemicals. The TRIzol ${ }^{\circledR}$ kit, BCA protein quantitative kit, SYBR Green PCR kit and reverse transcription kit used in the study were bought from the Thermo Fisher Scientific, Inc. The ECL kit (cat. no. WBKLS0100) was purchased from EDM Millipore. The primary antibodies for PPM1A and
Table I. Composition of Jiawei-Maxing-Shigan decoction.

Herbal medicines

Weight (g)

Roasted ephedra (Herba Ephedrae)

9

Gypsum (Gypsum Fibrosum)

Paeoniae radix (Radix Paeoniae Rubra)

18

Apricot seed (Semen Armeniacae Amarum)

12

Mulberry (Cortex mori Radicis)

12

Honeysuckle (Flos Lonicerae Japonicae)

12

Licorice (Radix Et Rhizoma Glycyrrhizae)

phosphorylated (p-)Smad2/3 were purchased from Abcam. The primary antibodies for Smad2/3, p-Smad2/3, E-cadherin, vimentin, $\alpha$-SMA and glyceraldehyde-3-phosphate dehydrogenase (GAPDH) were purchased from Cell Signaling Technology, Inc. PLKO.1, psPAX2 and pMD2G were acquired from Addgene, Inc. DH5 $\alpha$ competent cells were purchased from Beijing Transgen Biotech Co., Ltd.; 293T cells, from ATCC and pLVX-Puro from Clontech Laboratories, Inc.

Preparation of the JMSD-medicated serum and control serum. Wistar rats were randomly divided into the JMSD and control groups ( $\mathrm{n}=3$ per group). In the rats in the control and JMSD groups, $4 \mathrm{ml}$ of $0.9 \%$ sodium chloride and JMSD were intragastrically administered twice per day for 3 days, respectively. At $2 \mathrm{~h}$ after the last administration, the rats were euthanized with intraperitoneal injections of pentobarbital sodium $(100 \mathrm{mg} / \mathrm{kg})$ and a blood sample $(\sim 6 \mathrm{ml})$ was drawn from the abdominal aorta, pooled and clotted for $2 \mathrm{~h}$ at room temperature. Serum was isolated carefully by centrifuging the clotted blood at $2,000 \times \mathrm{g}$ for $20 \mathrm{~min}$ at $4^{\circ} \mathrm{C}$ and stored at $-70^{\circ} \mathrm{C}$ until use. In accordance with previous literature, the dose for preparation of medicated serum can be calculated as: Dose $=$ clinical dose $\mathrm{x}$ animal equivalent dose $\mathrm{x}$ dilution (15). In the present study, the animals were given $15 \mathrm{x}$ adult dose to prepare the JMSD-medicated serum. Primary type II AECs were treated the JMSD-medicated serum at doses of $\sim 2-10 \%$, which was diluted to $\sim 2 / 100-10 / 100$. Therefore, the dose used in this present study was not a very high dose. During the experiments, animal health and behavior were monitored daily to minimize suffering and distress. Greater than $20 \%$ weight loss, dehydration, or loss of ability to ambulate, were the signs we used to determine the time at which the animals should be euthanized. Confirmation of death was evaluated with vital signs including heart beats, pupillary response and respiratory pattern. No animals showed signs of humane endpoints and no rats were dead prior to the end of the experiments.

Preparation of type II AECs and cell culture. Primary type II AECs were prepared from SD rats as previously described (10); after the rats were euthanized with intraperitoneal injections of pentobarbital sodium $(100 \mathrm{mg} / \mathrm{kg})$. Dispase was instilled into the lung via a tracheal catheter for $15 \mathrm{~min}$ at $37^{\circ} \mathrm{C}$. The lungs were removed, carefully teased apart and treated with DNase I for $5 \mathrm{~min}$ at $37^{\circ} \mathrm{C}$. The cell suspension was passed through 150 , 15 and $7.5 \mu \mathrm{m}$ metal strainers and then centrifuged at $100 \mathrm{x} \mathrm{g}$ at $4^{\circ} \mathrm{C}$ for $8 \mathrm{~min}$. The cell pellet was resuspended in Dulbecco's 
Table II. Identification of constituents from Jiawei-Maxing-Shigan decoction.

\begin{tabular}{lll}
\hline Compound & RT/min & \multicolumn{1}{c}{ Herbal medicines } \\
\hline 1. Ephedrine hydrochloride & 0.56 & Honey-fried Herba Ephedrae \\
2. Levistilide A & 0.68 & Gypsum Fibrosum \\
3. Liquiritigenin & 1.01 & Radix Et Rhizoma Glycyrrhizae \\
4. Pseudoephedrine hydrochloride & 3.18 & Honey-fried Herba Ephedrae \\
5. Paeoniflorin & 5.09 & Radix Paeoniae Rubra \\
6. Astilbin & 5.70 & Cortex mori from Morus alba L. \\
7. Cycloastragenol & 5.10 & Radix Et Rhizoma Glycyrrhizae \\
8. Diosgenin & 5.74 & Stir-baked Semen Armeniacae Amarum \\
9. Rosmarinic acid & 6.32 & Flos Lonicerae Japonicae \\
10. Paeonol & 7.54 & Radix Paeoniae Rubra \\
\hline
\end{tabular}

modified Eagle's medium (DMEM) and plated to a culture dish precoated with rat IgG. After $1 \mathrm{~h}$ of culture, the non-adherent cells were collected and plated to another culture dish. After culturing for another $20 \mathrm{~min}$, the non-adherent cells were centrifuged at $100 \times \mathrm{g}$ at $4^{\circ} \mathrm{C}$ for $8 \mathrm{~min}$. The cell pellet was resuspended in DMEM containing $20 \%$ fetal bovine serum (HyClone; Cytiva) and cultured at $37^{\circ} \mathrm{C}$ in a $5 \% \mathrm{CO}_{2}$ incubator. The purity of the isolated cells was $>90 \%$ as determined with nitroblue tetrazolium/5-bromo-4-chloro-3-indolyl phosphate staining.

Constructions of the overexpression and low expression level of lentivirus. A lentivirus was constructed commercially by Genewiz, Inc. Briefly, the plasmid vectors were extracted and subsequently co-transfected into 293T cells with the packaging plasmids using Lipofectamine ${ }^{\circledR} 2000$ (Invitrogen; Thermo Fisher Scientific, Inc.). After $72 \mathrm{~h}$ of culture at $37^{\circ} \mathrm{C}$, supernatant of $293 \mathrm{~T}$ cells was collected and filtered using a $0.45-\mu \mathrm{m}$ filter. The short interfering (si)RNA sequences used are as follows: siPPM1A-1: 5'-CCAAGUGGACUUGAG ACAUUU-3'; siPPM1A-2: 5'-GCCUGAAGUCCAUGAUAU UUU-3'; siPPM1A-3': 5'-CCUUGAGAAAGUUUGCAA UUU-3'; and siNC: 5'-CAGUACUUUUGUGUAGUACAA-3'.

Cell treatment. Primary type II AECs were divided into four groups. Groups 1 and 2 were cultured with $10 \%$ serum collected from control rats. The cells in group 3 were incubated with $2 \% \mathrm{JMSD}$-medicated serum $(\mathrm{J})$ and $8 \%$ normal control rat serum $(\mathrm{N})$. Those in group 4 were incubated with $6 \% \mathrm{~J}$ serum and $4 \% \mathrm{~N}$ serum, whereas those in group 5 were incubated with $10 \% \mathrm{~J}$ serum. Groups $2-5$ were stimulated with 8 Gy of 60Co $\gamma$-rays (Hangzhou Cancer Hospital) at $3.64 \mathrm{~Gy} / \mathrm{min}$. After $24 \mathrm{~h}$ of culture, reverse transcription-quantitative (RT-q) PCR assay and western blotting analyses were performed.

$R T-q P C R$. Total RNA was isolated from cells with TRIzol ${ }^{\circledR}$ and subsequently reversed transcribed into cDNA with RevertAid First Strand cDNA Synthesis Kit (Thermo Fisher Scientific, Inc.) according to the manufacturer's protocol. Then, a real-time PCR analysis was performed using an ABI 7300 instrument (Applied Biosystems; Thermo Fisher Scientific, Inc.) at the following parameters: $95^{\circ} \mathrm{C}$ for $10 \mathrm{~min}$, followed by 40 cycles of annealing at $95^{\circ} \mathrm{C}$ for $15 \mathrm{sec}$ and amplification at $60^{\circ} \mathrm{C}$ for $45 \mathrm{sec}$. GAPDH was used as an internal control. The primers used were: PPM1A: 5'-TGCCAAATACTGCTGTGAG-3' (forward), 5'-CTGTTG ACCCACTTCTATCTG-3' (reverse); and GAPDH: 5'-GGA GTCTACTGGCGTCTTCAC-3' (forward), 5'-ATGAGCCCT TCCACGATGC-3' (reverse). The mRNA level in each sample was evaluated using the ${ }^{\Delta \Delta} \mathrm{CT}$ method (16). The experiments were repeated three times.

Western blotting assay. The proteins in the cells were prepared using a radioimmunoprecipitation assay lysis buffer (Beyotime Institute of Biotechnology) and the protein concentrations were measured using the bicinchoninic acid method. Equal amounts of protein $(30 \mu \mathrm{g})$ were loaded on $10 \%$ sodium dodecyl sulfate-polyacrylamide gel and then transferred onto a nitrocellulose membrane and blocked with $5 \%$ skimmed milk at room temperature for $1 \mathrm{~h}$. The membranes were then incubated with primary antibodies and subsequently incubated with HRP-conjugated secondary antibodies at room temperature for $1 \mathrm{~h}$. Lastly, the targeting bands were detected using ECL reagents. The primary antibodies were anti-PPM1A (cat. no. ab154489, 1:1,000) and anti-p-Smad2/3 (cat. no. ab63399, 1:1,000), purchased from Abcam. Anti-Smad2/3 (cat. no. 5678, 1:1,000), anti-E-cadherin (cat. no. 14472, 1:1,000), anti-vimentin (cat. no. 5741, 1:1,000), anti- $\alpha$-SMA (cat. no. 19245, 1:5,000) and anti-GAPDH (cat. no. 5174, 1:1,000) were obtained from Cell Signaling Technology, Inc. Densitometry was performed using ImageJ software (version 1.48; National Institutes of Health) with GAPDH as the loading control.

Statistical analysis. All experimental data are shown in mean \pm standard deviation. One-way ANOVA followed by Sidak's post hoc test was performed to analyze statistical comparisons. $\mathrm{P}<0.05$ was considered to indicate a statistically significant difference.

\section{Results}

Identification of 10 compounds. The aqueous extract from the JMSD was measured with high-performance liquid chromatography coupled with electrospray mass spectrometry 
A

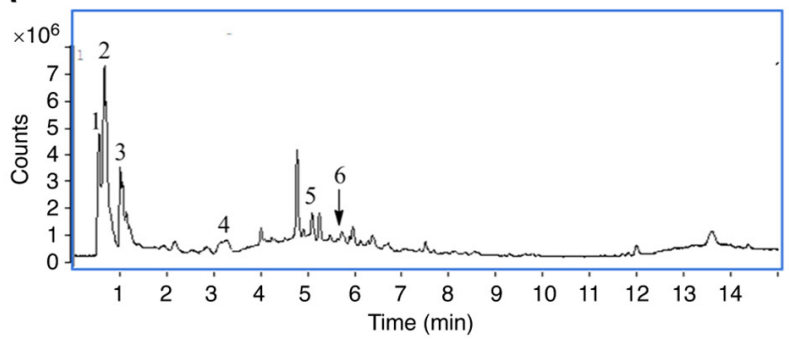

B

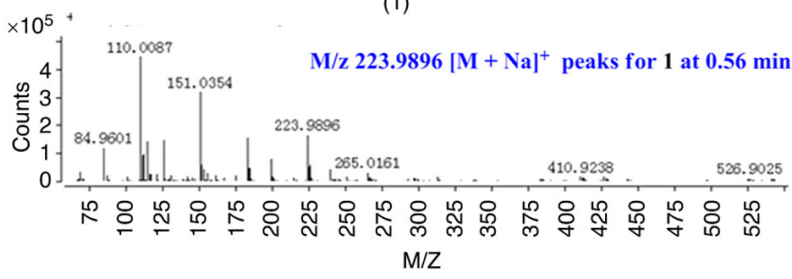

(3)

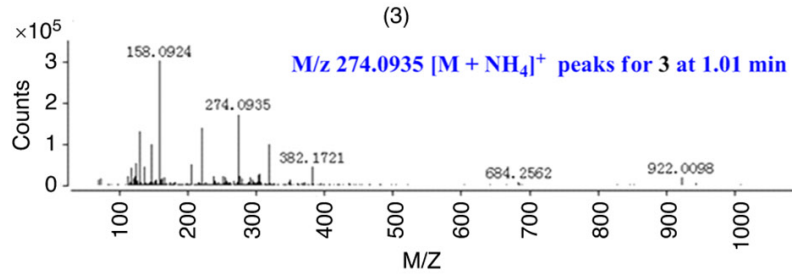

(5)

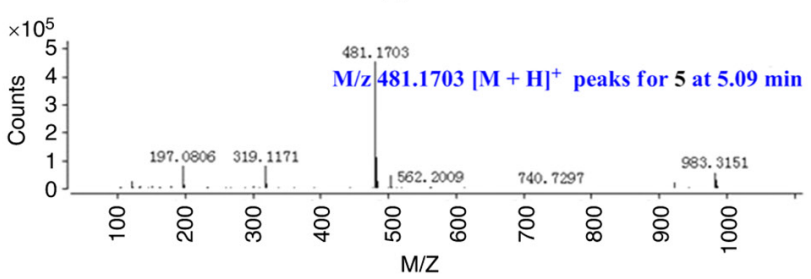

(7)

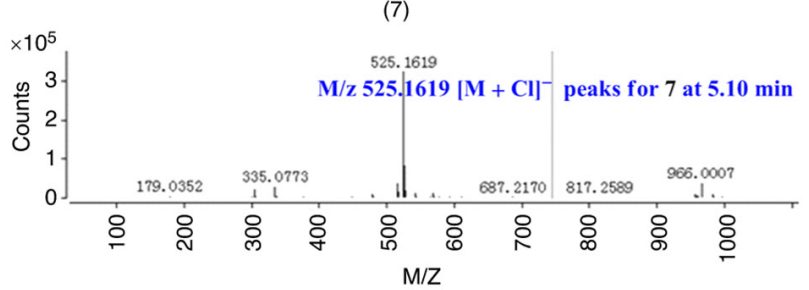

(9)

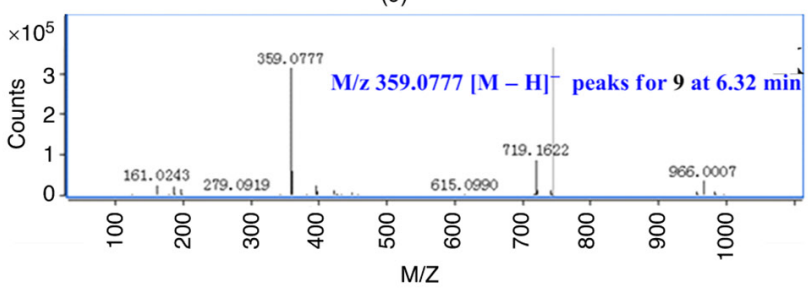

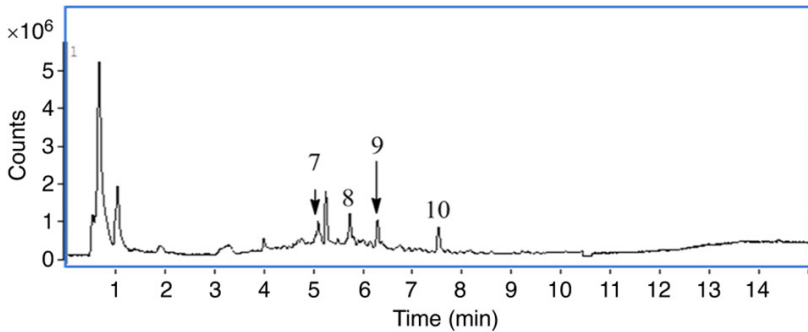

(2)

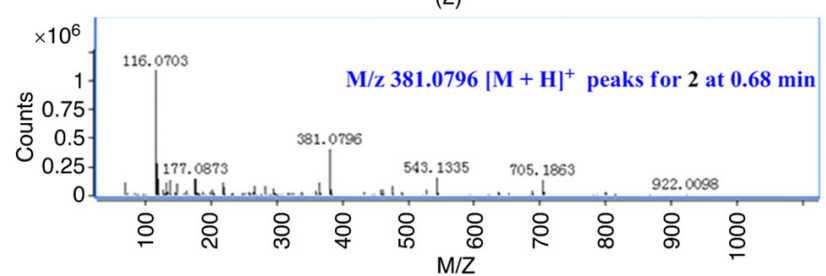

(4)

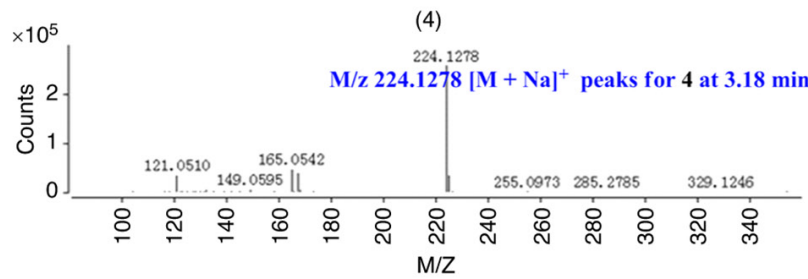

(6)

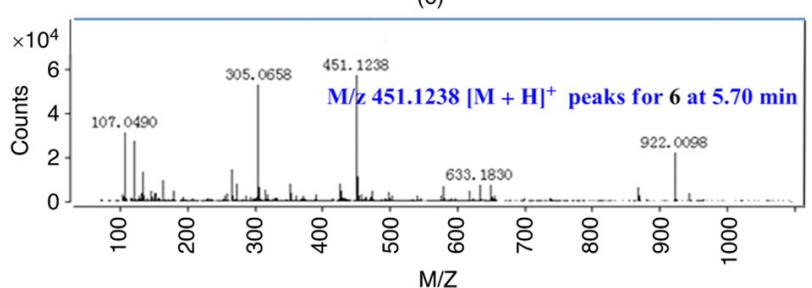

(8)

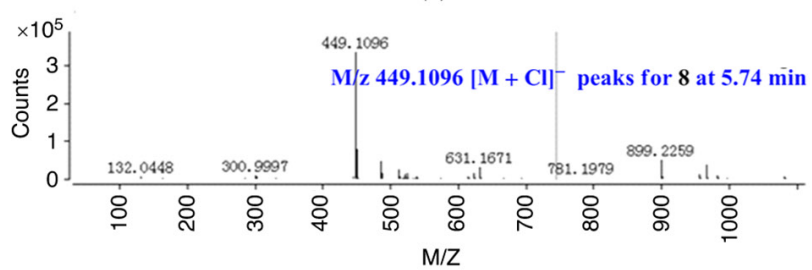

(10)

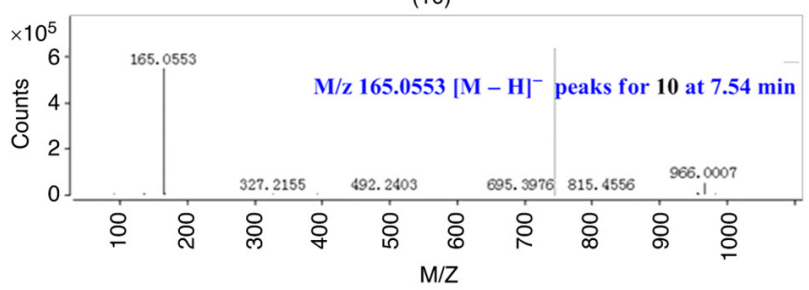

Figure 1. Screening the components in the aqueous extract of Jiawei-Maxing-Shigan decoction. (A) High-performance liquid chromatography coupled with electrospray mass spectrometry chromatogram of the aqueous extract in positive and negative modes. (B) The product ion mass spectra of (1) ephedrine hydrochloride, (2) levistilide A, (3) liquiritigenin, (4) pseudoephedrine hydrochloride, (5) paeoniflorin, (6) astilbin, (7) cycloastragenol, (8) diosgenin, (9) rosmarinic acid and (10) paeonol. Conditions: collision gas, ultrahigh-purity helium (He); nebulizer gas (N2), 35 psi; drying gas (N2), 10 L/min; drying temperature, $350^{\circ} \mathrm{C} ; \mathrm{HV}, 3500 \mathrm{~V}$; mass scan range, $100-2200 \mathrm{~m} / \mathrm{z}$; target mass, $500 \mathrm{~m} / \mathrm{z}$; compound stability, $100 \%$; and trap drive level, $100 \%$.

(HPLC/ESI-MS) in positive- and negative-ion modes (Fig. 1, Table II). The following 10 compounds were identified: ephedrine hydrochloride (1), levistilide A (2), liquiritigenin (3), pseudoephedrine hydrochloride (4), paeoniflorin (5), astilbin (6), cycloastragenol (7), diosgenin (8), rosmarinic acid (9) and paeonol (10), by comparing the retention times and MS data with the reference standards.

Decreased PPMIA expression level in type II AECs following radiation treatment. Following radiation treatment (60Co 


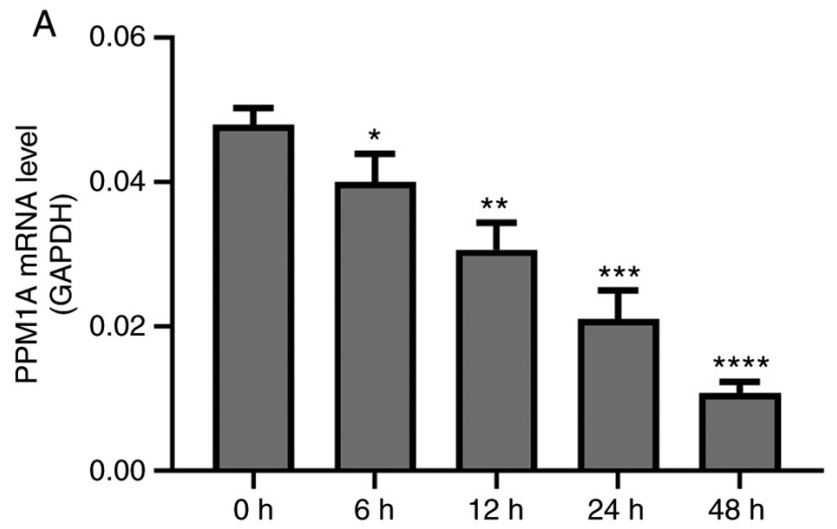

B
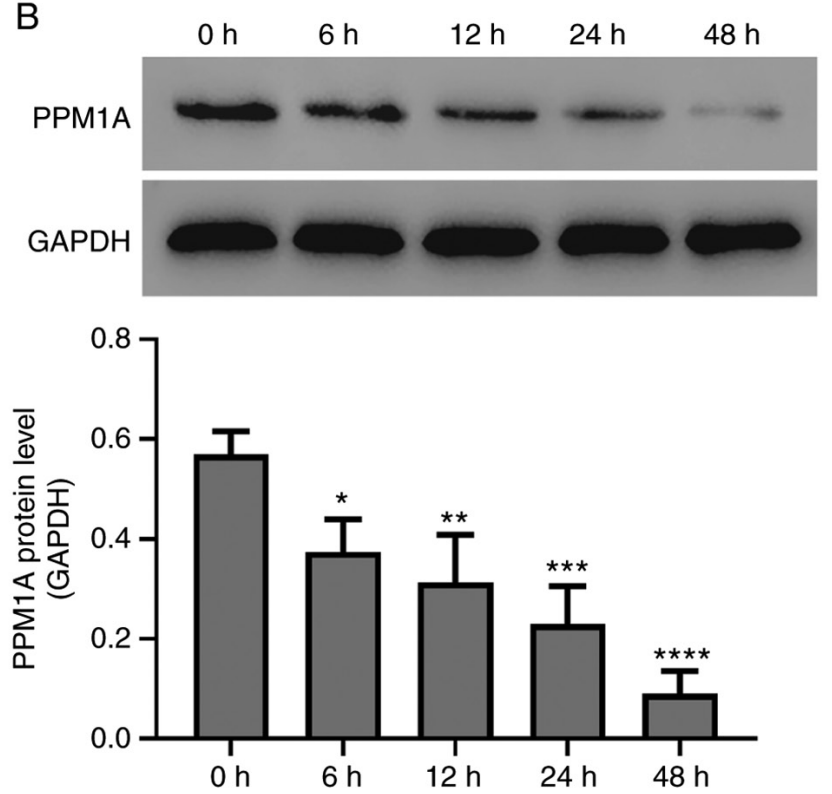

Figure 2. PPM1A expression level was downregulated in type II AECs following radiation treatment. Cells were treated with $8 \mathrm{~Gy}$ of ${ }^{60} \mathrm{Co} \gamma$-rays at a dose rate of $3.64 \mathrm{~Gy} / \mathrm{min}$ at room temperature. The (A) mRNA and (B) protein levels of PPM1A were detected with reverse transcription-quantitative PCR and western blotting, respectively. ${ }^{*} \mathrm{P}<0.05,{ }^{* *} \mathrm{P}<0.01,{ }^{* * * *} \mathrm{P}<0.001$ and ${ }^{* * * * *} \mathrm{P}<0.0001$, vs. 0 h. PPM1A, protein phosphatase $\mathrm{Mg}^{2+} / \mathrm{Mn}^{2+}$-dependent $1 \mathrm{~A}$.

$\gamma$-ray at 8 Gy), the PPM1A expression level in type II AECs was determined using RT-PCR and western blotting assays. As shown in Fig. 2, the results indicated that the radiation treatment could decrease the PPMIA expression levels in AECs both in terms of mRNA and protein expression levels $(\mathrm{P}<0.05)$ in an obvious time-dependent manner.

Attenuation of radiation-mediated decreased PPMIA expression level in type II AECs by JMSD-medicated serum. Furthermore, the effects of the JMSD-medicated serum on the PPM1A expressions in radiation-induced type II AECs were determined using RT-PCR and western blotting assays. The results shown in Fig. 3 suggested that JMSD-medicated sera (2, 6 and 10\%) could upregulate the PPM1A expressions as compared with the vehicle $(\mathrm{P}<0.05, \mathrm{P}<0.001$ and $\mathrm{P}<0.001$, respectively) in a concentration-dependent manner.

Attenuation of radiation-induced Smad activation and epithelial-mesenchymal transition by PPM1A overexpression. Consequently, PPM1A overexpressed AECs (Fig. 4A and B)
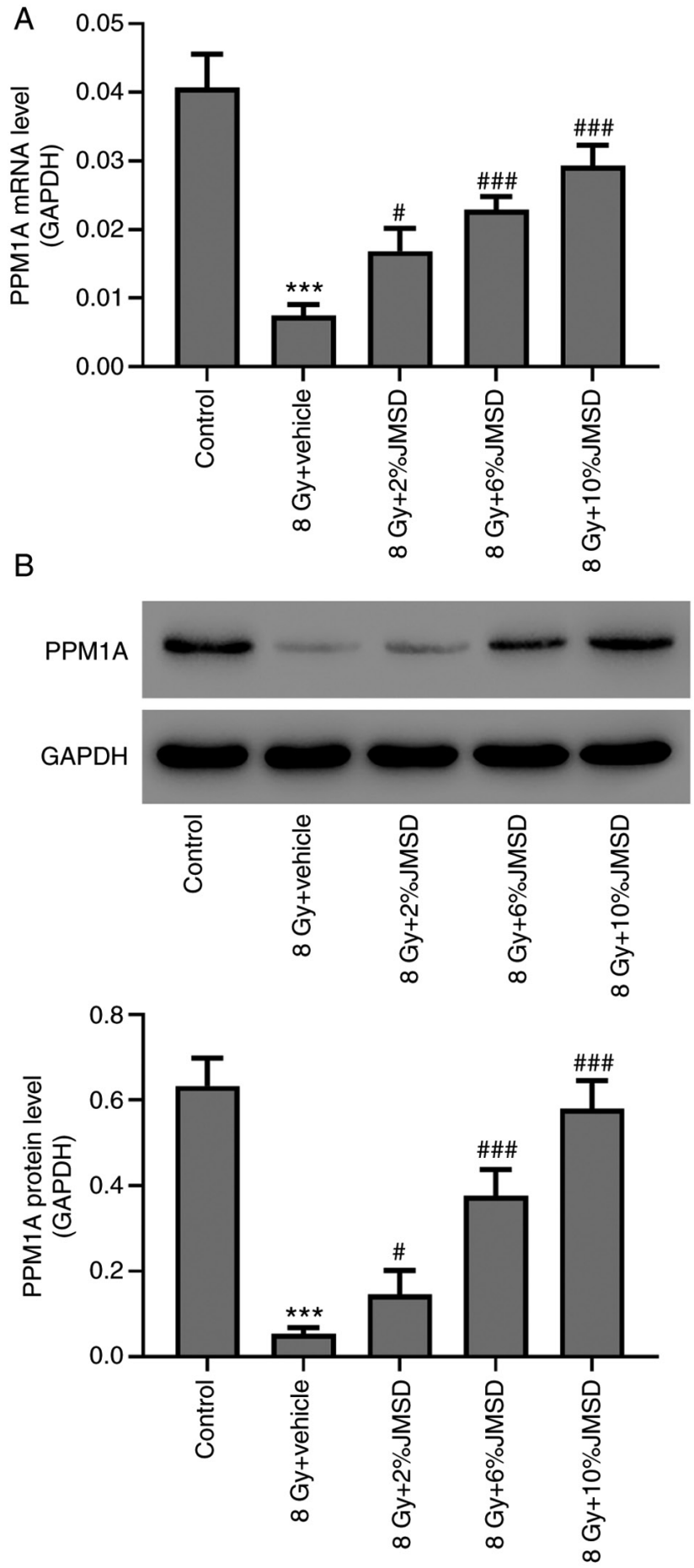

Figure 3. JMSD-medicated serum attenuated the radiation-mediated inhibition of PPM1A expression in type II alveolar epithelial cells. Cells were incubated with 0,2, 6 and 10\% JSMD-medicated serum and then treated with $8 \mathrm{~Gy}$ of ${ }^{60} \mathrm{Co} \gamma$-rays. After $24 \mathrm{~h}$, the (A) mRNA and (B) protein levels of PPM1A were detected with reverse transcription-quantitative PCR and western blotting, respectively. ${ }^{* * *} \mathrm{P}<0.001$, vs. the control; ${ }^{\#} \mathrm{P}<0.05$, $\# \# \mathrm{P}<0.001$, vs. 8 Gy + vehicle. JMSD, Jiawei-Maxing-Shigan decoction; PPM1A, protein phosphatase $\mathrm{Mg}^{2+} / \mathrm{Mn}^{2+}$-dependent $1 \mathrm{~A}$.

were constructed to investigate the role of PPM1A. The results showed that compared with vector expression, the PPM1A and E-cadherin expressions were upregulated, whereas the p-Smad2/3, vimentin and $\alpha$-SMA expressions were downregulated in the PPM1A-overexpressed AECs (Fig. 4C). Morphology analysis showed that elongated spindle-like 


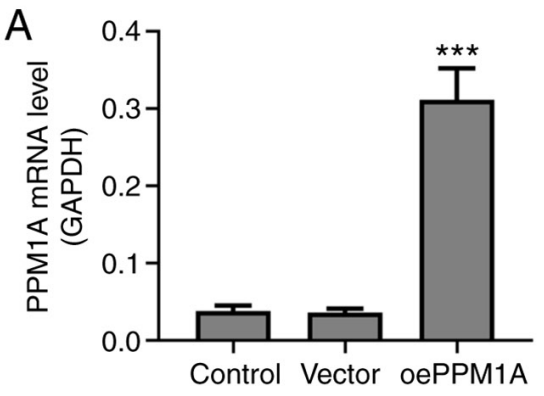

C

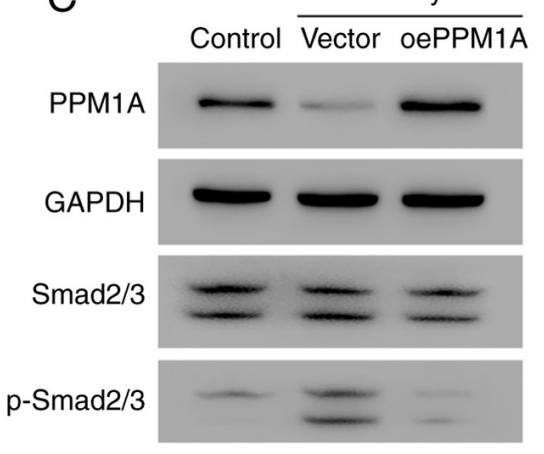

GAPDH

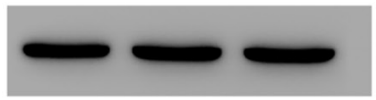

B

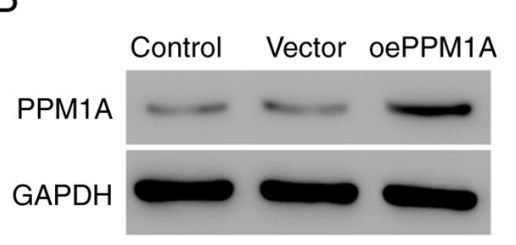

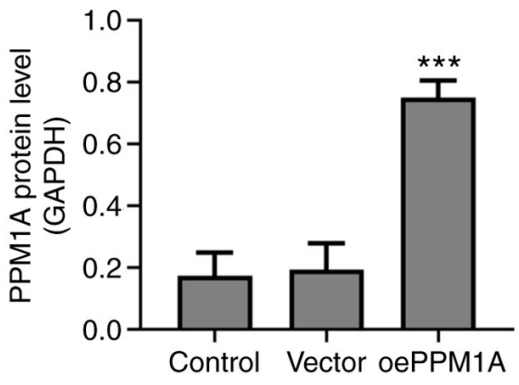

8 Gy
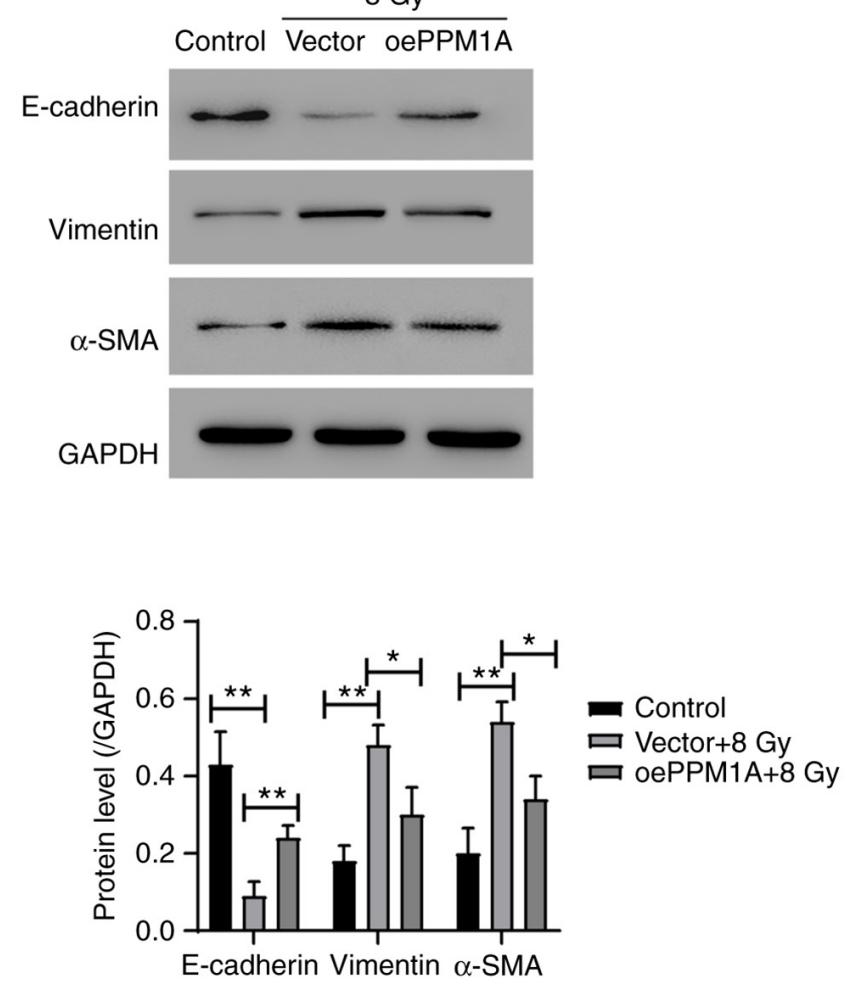

8 Gy

$\mathrm{D}$

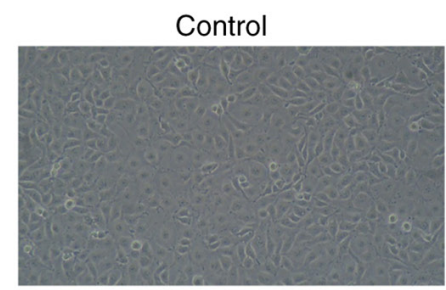

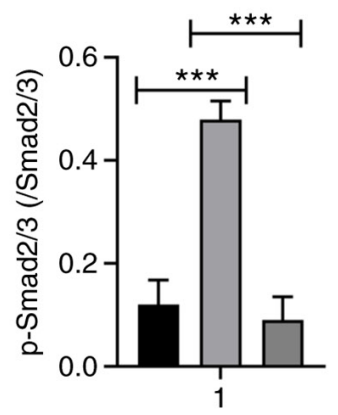

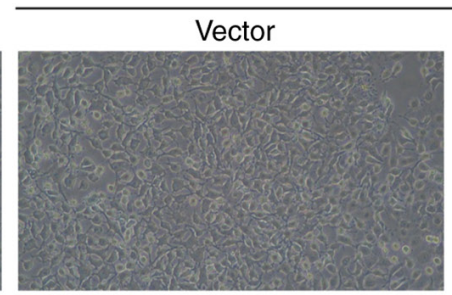

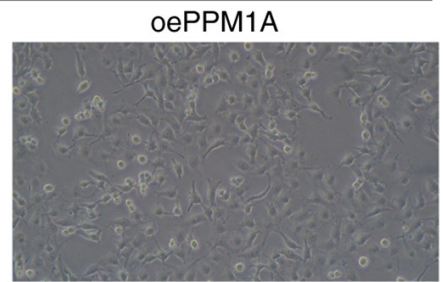

Figure 4. PPM1A overexpression attenuated the radiation-induced Smad activation and epithelial-mesenchymal transition. Type II AEC was transduced with oePPM1A. After $24 \mathrm{~h}$, the (A) mRNA and (B) protein levels of PPM1A were detected using reverse transcription-quantitative PCR and western blotting, respectively. ${ }^{* * *} \mathrm{P}<0.001$, vs. vector. Type II AECs were transduced with oePPM1A and then treated with $8 \mathrm{~Gy}$ of ${ }^{60} \mathrm{Co} \gamma$-rays. After $24 \mathrm{~h}$, the protein levels were measured using (C) western blotting and (D) phase contrast images were obtained (magnification, $\mathrm{x} 200$ ). ${ }^{*} \mathrm{P}<0.05,{ }^{* * *} \mathrm{P}<0.01,{ }^{* * * *} \mathrm{P}<0.001$, vs. vector. PPM1A, protein phosphatase $\mathrm{Mg}^{2+} / \mathrm{Mn}^{2+}$-dependent 1A; AEC, alveolar epithelial cells; oe, overexpression; $\mathrm{p}$-, phosphorylated; $\alpha$-SMA, $\alpha$-smooth muscle actin.

type cells were increased in radiation-treated cells, and such morphological alteration was decreased when PPM1A was overexpressed (Fig. 4D).

Attenuation of radiation-induced Smad activation and epithelial-mesenchymal transition by JMSD-medicated serum via the regulation of PPMIA expression. Subsequently, the role of PPM1A was further studied using the siRNA interference of PPM1A. The present study designed three siRNA sequences for the PPM1A and siNC, a scrambled sequence, was used as a negative control. siPPM1A-2 was selected as the siRNA sequence for the following studies (Fig. 5A and B).

Furthermore, the results suggested that compared with the cells in the vehicle group, the PPM1A siRNA-interfered AECs showed downregulated PPM1A and E-cadherin expressions but upregulated $\mathrm{p}-\mathrm{Smad} 2 / 3$, vimentin and $\alpha$-SMA expressions (Fig. 5C). In addition, after treatment with SB431542, a TGF- $\beta 1 /$ Smad signaling inhibitor, the $\mathrm{p}-\mathrm{Smad} 2 / 3$, vimentin and $\alpha$-SMA expression levels were decreased, whereas the E-cadherin expression level was increased in the PPM1A 

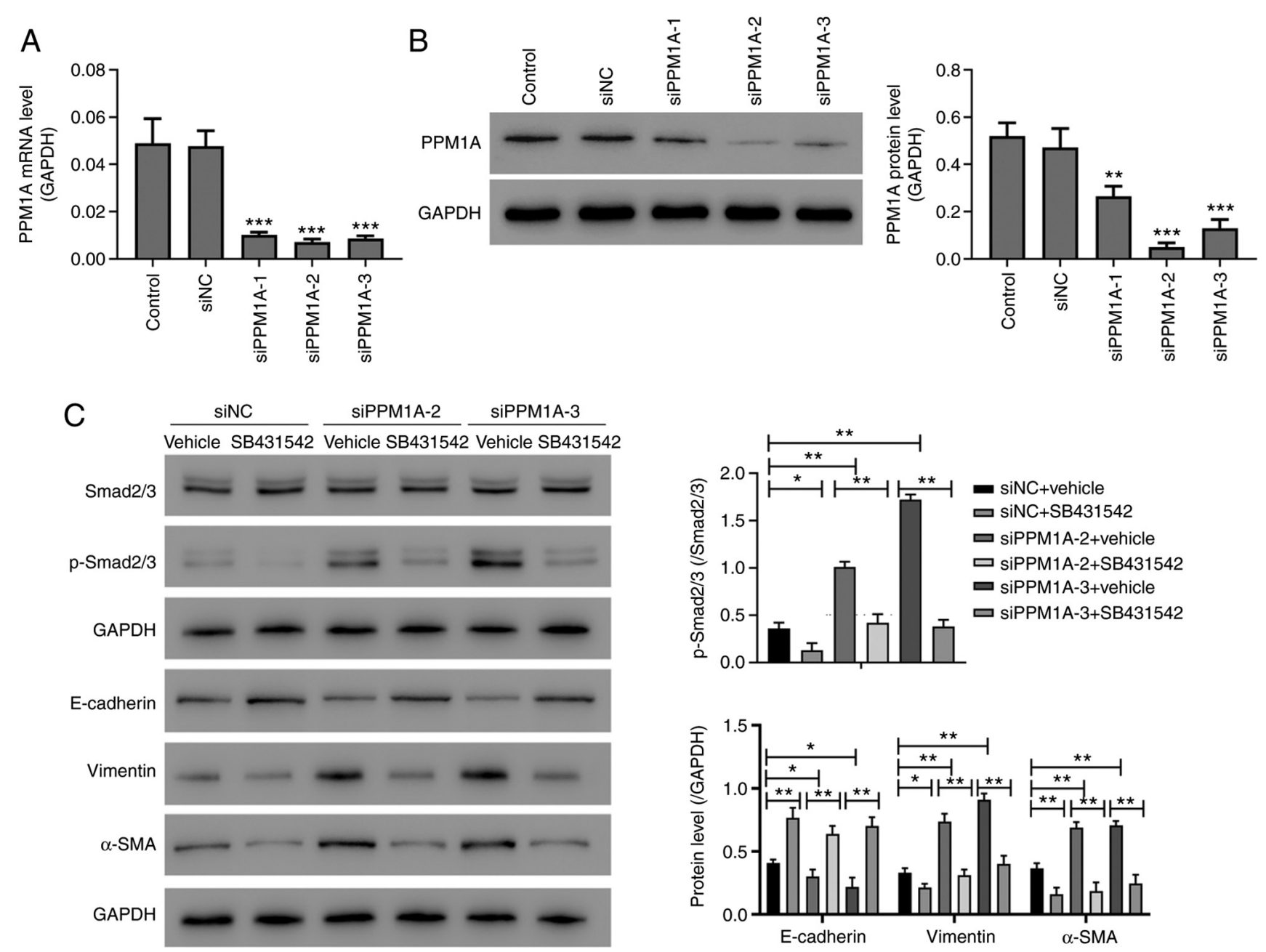

Figure 5. PPM1A knockdown promoted the epithelial-mesenchymal transition by activating the TGF- $\beta 1 /$ Smad pathway. (A and B) Type II AECs were transduced with siPPM1A. After $24 \mathrm{~h}$, the mRNA (A) and protein levels (B) of PPM1A were detected with RT-qPCR and western blotting, respectively. ${ }^{* * *} \mathrm{P}<0.001$ vs. siNC. (C) Type II AECs were transduced with siPPM1A and then treated with $10 \mu \mathrm{mol} / 1 \mathrm{TGF}-\beta 1 / \mathrm{Smad}$ inhibitor (SB431542). After $24 \mathrm{~h}$, the protein levels were measured using western blotting. ${ }^{*} \mathrm{P}<0.05,{ }^{* * *} \mathrm{P}<0.01,{ }^{* * * *} \mathrm{P}<0.001$. PPM1A, protein phosphatase $\mathrm{Mg}^{2+} / \mathrm{Mn}^{2+}$-dependent $1 \mathrm{~A}$; AEC, alveolar epithelial cells; si, short interfering; p-, phosphorylated; $\alpha$-SMA, $\alpha$-smooth muscle actin; NC, negative control.

knockdown AECs as compared with the vehicle group. Combined with the above-mentioned results, it was hypothesized that PPM1A might be a crucial target for EMT in AECs in TGF- $\beta 1 /$ Smad signaling.

Finally, the role of PPM1A in the attenuating effects of JMSD against radiation-induced EMT was studied in primary type II VECs. The image depicted in Fig. 6 suggested that JMSD-medicated serum could increase the E-cadherin expression level and decrease the $\mathrm{p}-\mathrm{Smad} 2 / 3$, vimentin and $\alpha$-SMA expression levels. However, these effects could be blocked by siPPM1A-2. Consequently, it is hypothesized that PPM1A-2 is a crucial target for the anti-EMT effect of JMSD in VECs.

\section{Discussion}

Herbal medicines are important alternative and complementary remedies for the treatment of various diseases, particularly those that cannot be treated with western drugs. Radiation-induced pulmonary fibrosis is an intractable disease in clinical practice and an increasing number of researchers have attempted to search for treatments for pulmonary fibrosis from herbal medicines (17). Some extracts/compounds from natural herbal medicines are feasible for treating lung pulmonary diseases, such as polydatin from Polygonum cuspidatum $(18,19)$ and astragaloside IV from Astragalus membranaceus (20,21). JMSD administration can attenuate the radiation-induced EMT in AECs via the TGF- $\beta /$ Smad signaling (7). The present study on JMSD against radiation-induced EMT found that PPM1A exhibits a crucial role in its anti-EMT activity.

TGF- $\beta /$ Smad signaling serves a key effect in the EMT process of AECs and further induction of pulmonary fibrosis (22). Under radiation, the TGF- $\beta 1$ expression level in bronchoalveolar lavage fluids can be increased and then, TGF- $\beta /$ Smad signaling is activated (23). First, TGF- $\beta$ expression can promote the phosphorylation of $\mathrm{Smad} 2 / 3$ by binding to the TGF- $\beta$ receptor in the cell membrane and then the transcription of $\mathrm{p}-\mathrm{Smad} 2 / 3$ to the cell nucleus, resulting in some transcriptional regulation of a number of genes (24). Consequently, some of the responding productions such as Snail, connective tissue growth factor and matrix metalloproteinases would be released and further result in EMT (25). PPM1A is a phosphatase of the serine/threonine PPM family and the main substrates of PPM1A include MAPK, Smad2 

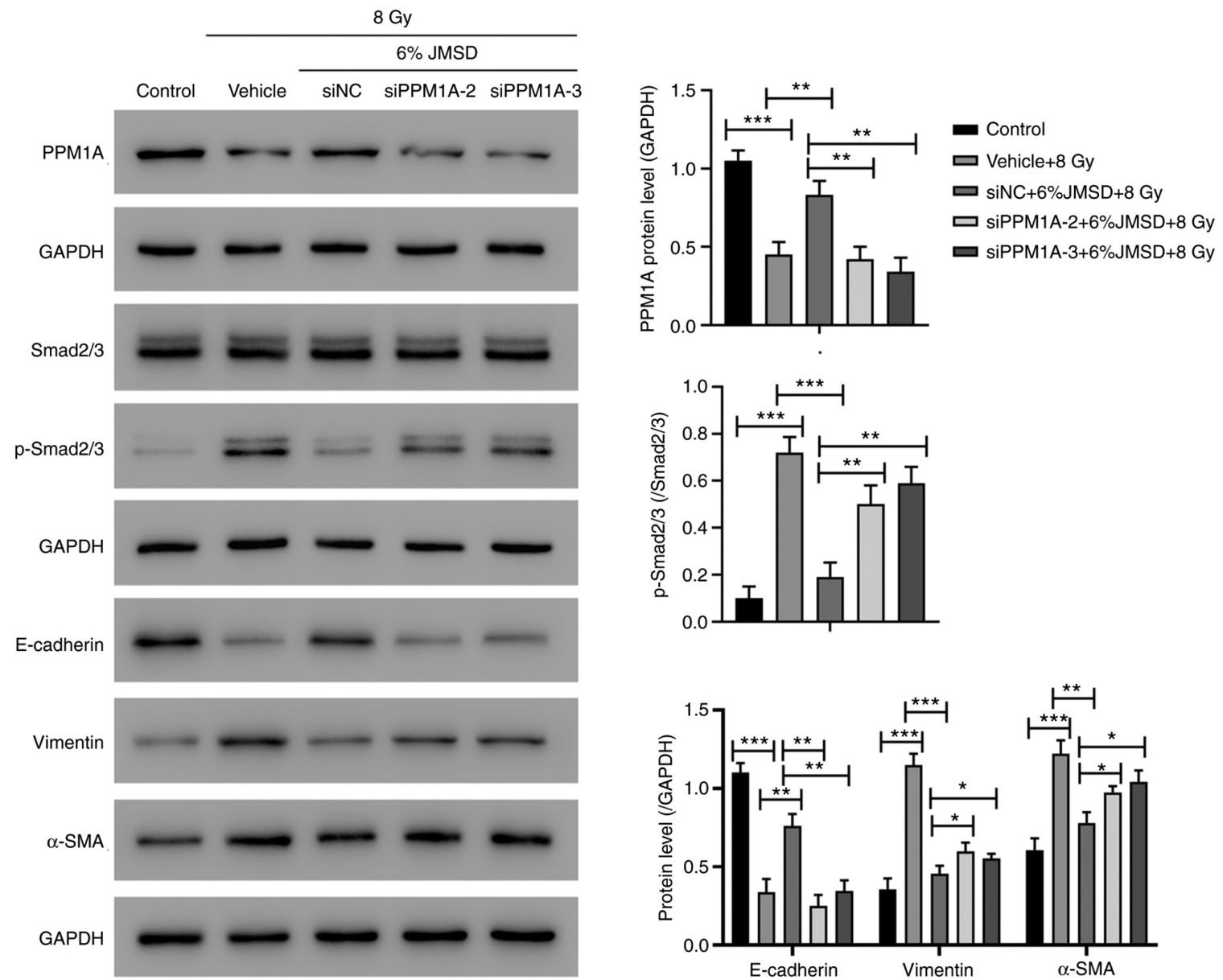

Figure 6. JMSD-medicated serum attenuated radiation-induced Smad activation and epithelial-mesenchymal transition by regulating the PPM1A expression. Type II AECs, which were incubated with $6 \%$ JSMD-medicated serum, were transduced with siPPM1A (or siNC) and then treated with 8 Gy of ${ }^{60} \mathrm{Co} \gamma$-rays. After $24 \mathrm{~h}$, the protein levels were measured using western blotting. " $\mathrm{P}<0.05,{ }^{* *} \mathrm{P}<0.01,{ }^{* * * *} \mathrm{P}<0.001$. JMSD, Jiawei-Maxing-Shigan decoction; PPM1A, protein phosphatase $\mathrm{Mg}^{2+} / \mathrm{Mn}^{2+}$-dependent $1 \mathrm{~A}$; AEC, alveolar epithelial cells; si, short interfering; NC, negative control; $\mathrm{p}$-, phosphorylated; $\alpha$-SMA, $\alpha$-smooth muscle actin.

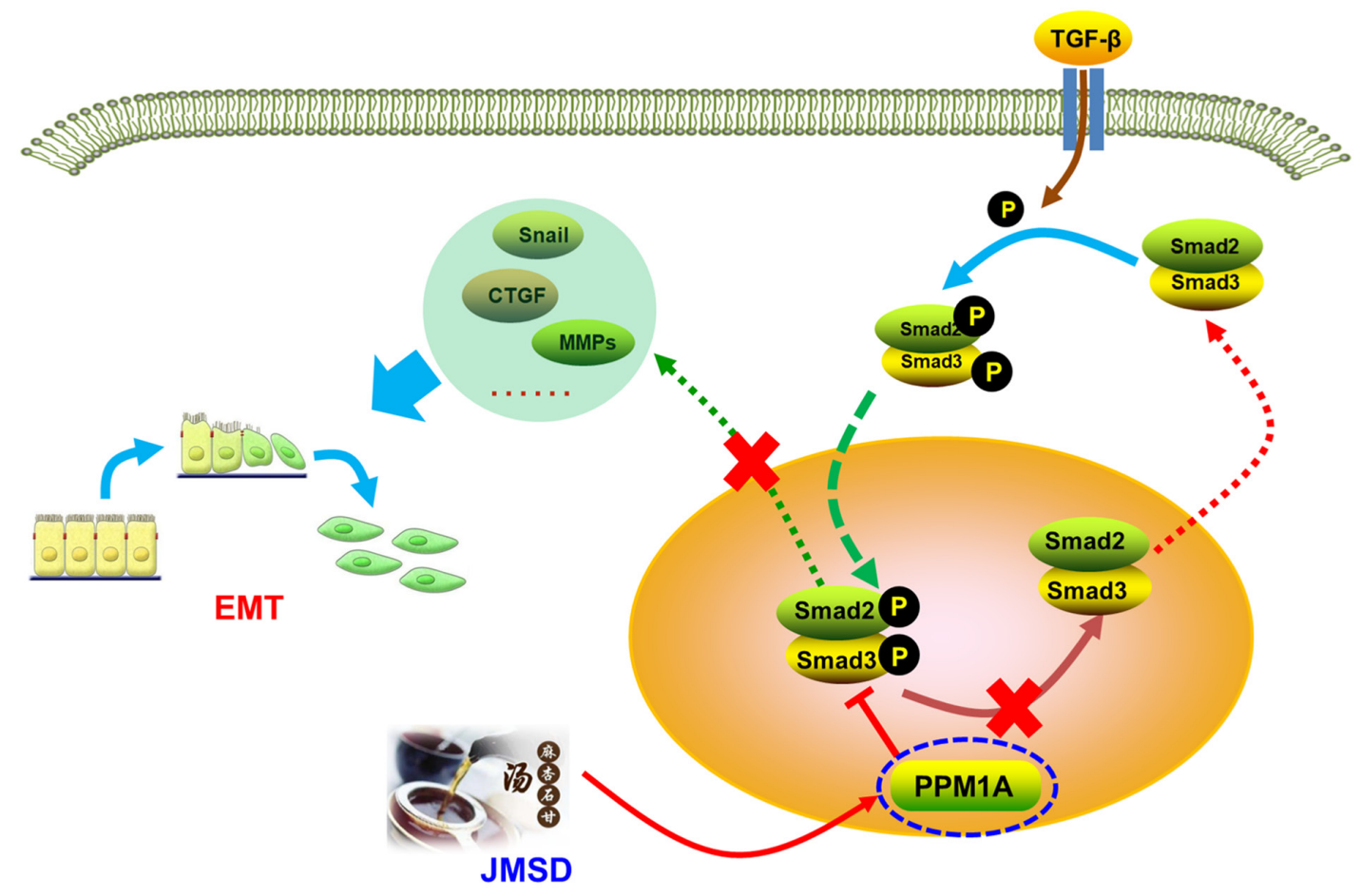

Figure 7. PPM1A is a key target of JMSD administration for attenuating the radiation-induced EMT in primary type II AECs. PPM1A, protein phosphatase $\mathrm{Mg}^{2+} / \mathrm{Mn}^{2+}$-dependent 1A; JMSD, Jiawei-Maxing-Shigan decoction; EMT, epithelial-mesenchymal transition; AECs, alveolar epithelial cells; p, phosphorylation. 
and Smad3 and MKKs (12-14). PPM1A serves an important role in the signal transduction of TGF- $\beta /$ Smad signaling and can inactivate TGF- $\beta /$ Smad signaling by dephosphorylating Smad2/3 (26,27) (Fig. 7). The present study found that PPM1A expression level was reduced in type II AECs following radiation treatment. PPM1A overexpression attenuated EMT, while PPM1A knockdown showed reverse effects on EMT. Furthermore, treatment with a TGF- $\beta 1 /$ Smad signaling inhibitor blocked PPM1A knockdown-induced EMT. Therefore, PPM1A upregulation is a potential strategy for treating EMT and pulmonary fibrosis through inhibiting TGF- $\beta 1 / \mathrm{Smad}$ signaling.

Previously, we have reported that JMSD attenuated radiation-induced EMT in AECs by regulating TGF- $\beta / \mathrm{Smad}$ signaling (7). The present study found that JMSD-medicated serum attenuated the radiation-mediated inhibition of PPM1A expression in type II AECs. The role of PPM1A in the anti-EMT effects of JMSD in AECs was then examined and it was found that JMSD treatment could increase the PPM1A and E-cadherin expression levels. JMSD also decreased the p-Smad2/3, vimentin and $\alpha$-SMA expressions in VECs. After knockdown of the PPM1A, the regulating effects of JMSD on these proteins were blocked. The data indicated that JMSD acted against radiation-induced EMT through upregulating PPM1A.

However, the present study has some limitations. It did not investigate the biological effects of the detailed components identified using HPLC/ESI-MS owing to the limited time. In addition, upstream targets of PPM1A should also be investigated in succeeding works. The research regarding JMSD is continuing and it is hoped to investigate the detail mechanisms by which JMSD increase the PPMIA expression and the biological effects of detail components of JMSD and present the related results in the future.

Together, the present study suggested that PPM1A is a key target of JMSD administration for the attenuation of the radiation-induced EMT in primary type II alveolar epithelial cells via TGF- $\beta 1 /$ Smad signaling.

\section{Acknowledgements}

Not applicable.

\section{Funding}

The present study was supported by Science Technology Department of Zhejiang Province (grant no. 2018C03025), Zhejiang Provincial Natural Science Foundation of China (grant no. LY15H290002) and Zhejiang Province Traditional Chinese Medicine Science and Technology Planning Project (grant no. 2021ZA099).

\section{Availability of data and materials}

The data used to support the findings of this study are available from the corresponding author upon request.

\section{Authors' contributions}

SL and JL conceived and designed the study, JL, ZL, XL, YS and JS collected and analyzed the data and SL and JL wrote the manuscript. All authors read and approved the final manuscript. SL and JL confirm the authenticity of all the raw data.

\section{Ethics approval and consent to participate}

The experimental protocols were approved by the Animal ethics committee of Zhejiang Chinese Medicine University (Hangzhou, China; approval no. 2019485).

\section{Patient consent for publication}

Not applicable.

\section{Competing interests}

The authors declare that they have no competing interests.

\section{References}

1. Tian S, Zhang X, Jiang R, Pillai RN, Owonikoko TK, Steuer CE, Saba NF, Pakkala S, Patel PR, Belani CP, et al: Survival outcomes with thoracic radiotherapy in extensive-stage small-cell lung cancer: A propensity score-matched analysis of the national cancer database. Clin Lung Cancer 20: 484-493.e6, 2019.

2. Rodrigues G and Movsas B: Future directions in palliative thoracic radiotherapy. Curr Opin Support Palliat Care 6: 91-96, 2012.

3. Simone CB II: Thoracic radiation normal tissue injury. Semin Radiat Oncol 27: 370-377, 2017.

4. Giuranno L, Ient J, De Ruysscher D and Vooijs MA: Radiation-Induced Lung Injury (RILI). Front Oncol 9: 877, 2019.

5. Ota C, Ng-Blichfeldt JP, Korfei M, Alsafadi HN, Lehmann M, Skronska-Wasek W, M De Santis M, Guenther A, Wagner DE and Königshoff M: Dynamic expression of HOPX in alveolar epithelial cells reflects injury and repair during the progression of pulmonary fibrosis. Sci Rep 8: 12983, 2018.

6. Nagarajan D, Wang L, Zhao W and Han X: Trichostatin A inhibits radiation-induced epithelial-to-mesenchymal transition in the alveolar epithelial cells. Oncotarget 8: 101745-101759, 2017.

7. Lu J, Zhong Y, Lin X, Lin Z, Chen Z, Wu X, Wang N and Lin S: Jiawei Maxing Shigan Decoction (JMSD) attenuates radiation-induced epithelial-mesenchymal transition of primary rat type II alveolar epithelial cells. Int J Clin Exp Med 10: 16292-16300, 2017.

8. Yan-He LI: Clinical effect of Jiawei Maxingshigan decoction treatment for pediatric asthma. Laboratory Medicine \& Clinic 2013.

9. Lin T and Geng R: To Observe the Curative Effect of Western Medicine in the Treatment Combined With Jiawei Maxingshigan Decoction in Treating Acute Attack of Chronic Bronchitis. China Continuing Medical Education 2015.

10. Li J: Clinical observation of Jiawei Maxing Shigan Decoction in complementary treatment of 48 cases of infantile mycoplasma pneumonia. J Pediatr Traditional Chin Med 3: 40-41, 2007 (In Chinese).

11. Wang J, Li YQ, Zhang WP, Zhang T and Zhou X: The effects of Jiawei Maxing Shigan Decoction on radioactive lung injury Tianjin J Traditional Chin Med 28: 377-378, 2011.

12. Geng J, Fan J, Ouyang Q, Zhang X, Zhang X, Yu J, Xu Z, Li Q, Yao X, Liu X and Zheng J: Loss of PPM1A expression enhances invasion and the epithelial-to-mesenchymal transition in bladder cancer by activating the TGF- $\beta /$ Smad signaling pathway. Oncotarget 5: 5700-5711, 2014.

13. Lammers T, Peschke P, Ehemann V, Debus J, Slobodin B, Lavi S and Huber P: Role of PP2Calpha in cell growth, in radio- and chemosensitivity, and in tumorigenicity. Mol Cancer 6: 64, 2007.

14. Mazumdar A, Tahaney WM, Reddy Bollu L, Poage G, Hill J, Zhang Y, Mills GB and Brown PH: The phosphatase PPM1A inhibits triple negative breast cancer growth by blocking cell cycle progression. NPJ Breast Cancer 5: 22, 2019.

15. Zhang JT, Wang P, Liu AF, Yang G, Yuan-Dong LI and Zhang C: Overview about preparation methods of serum containing Chinese medicine. China J Traditional Chin Med Pharm 2015.

16. Livak KJ and Schmittgen TD: Analysis of relative gene expression data using real-time quantitative PCR and the 2(-Delta Delta C(T)) method. Methods 25: 402-408, 2001. 
17. Ghasemian M, Owlia S and Owlia MB: Review of anti-inflammatory herbal medicines. Adv Pharmacol Sci 2016: 9130979 , 2016.

18. Peng W, Qin R, Li X and Zhou H: Botany, phytochemistry, pharmacology, and potential application of Polygonum cuspidatum Sieb.et Zucc.: A review. J Ethnopharmacol 148: 729-745, 2013.

19. Zeng H, Wang Y, Gu Y, Wang J, Zhang H, Gao H, Jin Q and Zhao L: Polydatin attenuates reactive oxygen species-induced airway remodeling by promoting Nrf2-mediated antioxidant signaling in asthma mouse model. Life Sci 218: 25-30, 2019.

20. Fu J, Wang Z, Huang L, Zheng S, Wang D, Chen S, Zhang H and Yang S: Review of the botanical characteristics, phytochemistry, and pharmacology of Astragalus membranaceus (Huangqi). Phytother Res 28: 1275-1283, 2014.

21. Qian W, Cai X, Qian Q, Zhang W and Wang D: Astragaloside IV modulates TGF- $\beta 1$-dependent epithelial-mesenchymal transition in bleomycin-induced pulmonary fibrosis. J Cell Mol Med 22: 4354-4365, 2018.

22. Lu J,Zhong Y, Chen J, Lin X, Lin Z, Wang N and Lin S: Radiation enhances the Epithelial-Mesenchymal transition of A549 cells via miR3591-5p/USP33/PPM1A. Cell Physiol Biochem 50: 721-733, 2018.
23. Barthelemy-Brichant N, Bosquée L, Cataldo D, Corhay JL, Gustin M, Seidel L, Thiry A, Ghaye B, Nizet M, Albert A, et al: Increased IL-6 and TGF-betal concentrations in bronchoalveolar lavage fluid associated with thoracic radiotherapy. Int J Radiat Oncol Biol Phys 58: 758-767, 2004.

24. Hu HH, Chen DQ, Wang YN, Feng YL, Cao G, Vaziri ND and Zhao YY: New insights into TGF-beta/Smad signaling in tissue fibrosis. Chem Biol Interact 292: 76-83, 2018.

25. Kasai H, Allen JT, Mason RM, Kamimura T and Zhang Z: TGF-betal induces human alveolar epithelial to mesenchymal cell transition (EMT). Respir Res 6: 56, 2005.

26. Xu F, Liu C, Zhou D and Zhang L: TGF- $/$ /Smad Pathway and its regulation in hepatic fibrosis. J Histochem Cytochem 64: 157-167, 2016.

27. Zhu F, Xie N, Jiang Z, Li G, Ma L and Tong T: The cellular Senescence-Inhibited gene is essential for PPM1A Myristoylation to modulate transforming growth factor $\beta$ signaling. Mol Cell Biol 38: e00414-18, 2018.

This work is licensed under a Creative Commons Attribution-NonCommercial-NoDerivatives 4.0 International (CC BY-NC-ND 4.0) License. 